\title{
Automated AFM Boosts Throughput in Automatic Defect Review
}

\author{
Ryan YK Yoo
}

Park Systems Inc. 3040 Olcott St., Santa Clara, CA 95054

ryoo@parkAFM.com

\section{Introduction}

The scaling trend in semiconductor design requires tighter control over defects on wafers. To characterize the defects, both inspection and review tools are needed. The inspection tools such as surface scanning inspection systems (SSIS) are employed to locate and map defect sites. Then review tools such as scanning electron microscopes (SEMs) are used to obtain morphological information.

Large defects, which are visible by light optical microscopy (LOM), can be easily found by an inspection tool and imaged by a review tool. However, as processing requirements become more stringent, the defect size of interest gets smaller, below the diffraction limit of LOM. To locate and identify small defects, a large survey scan needs to be performed by the review tool in order to find the exact location of the defects (Figure 1).

Defect review SEM has been used to image the defects of a bare wafer after defect sites are mapped by the laser-scattering defect inspection tools such as Surfscan from KLA-Tencor. However, the imaging by defect review SEM is limited to $2 \mathrm{D}$ and cannot provide $3 \mathrm{D}$ information, which has become more crucial in recent days for bare wafer manufacturing control. Also, smaller defects, less than $50 \mathrm{~nm}$, are increasingly difficult to detect by the defect review SEM because irradiation damage on the bare surface or small-sized defects can change morphologies.

Atomic force microscopy (AFM) is gaining more importance as a review tool because it can directly measure both defect dimensions (height and area) and certain physical properties of defects of interest such as hardness by monitoring its force versus distance curve (force being measured by deflection of an AFM probe) and various electrical properties by measuring electric field-deflection of a probe. However, for engineers and researchers working with bare wafers, the process of identifying nanoscale defects is time-consuming with conventional manual AFM, hindering productivity, let alone throughput. Thus, it is important to have an automated solution for failure analysis and production engineers, using a review tool. who need to find the cause of defect sites on bare wafers and minimize them.

To address this need, Park Systems has developed the automatic defect review (ADR) AFM, which speeds up and improves the way defects are imaged and analyzed. The commercial name for this device is the Park NX-Wafer. With this solution, users are now able to obtain additional details such as height/depth information of the defects, which was not possible or too expensive to obtain with SEM-based ADR. This article describes this AFM defect review method.

\section{Materials and Methods}

From inspection tool to review AFM. The general defect review concept is simple, yet it becomes complicated when done on the nanometer scale-simple, because all it would require is the proper transfer of the defect map from an inspection tool and relocating those same defects

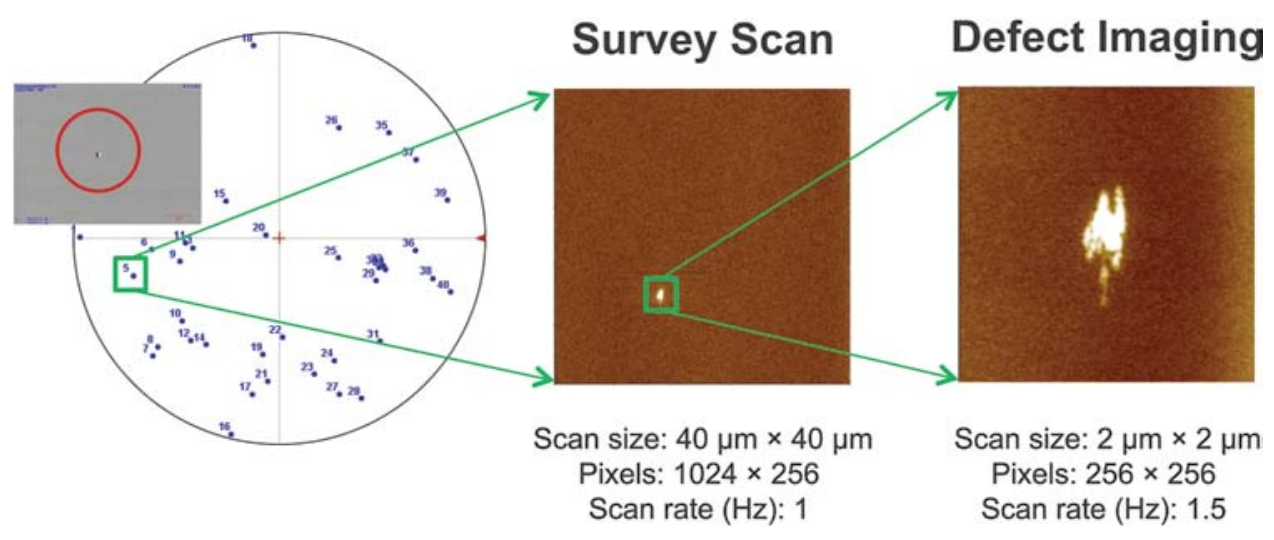

Figure 1: The goal of defect review is to locate the defect site identified by an inspection tool and image the defect

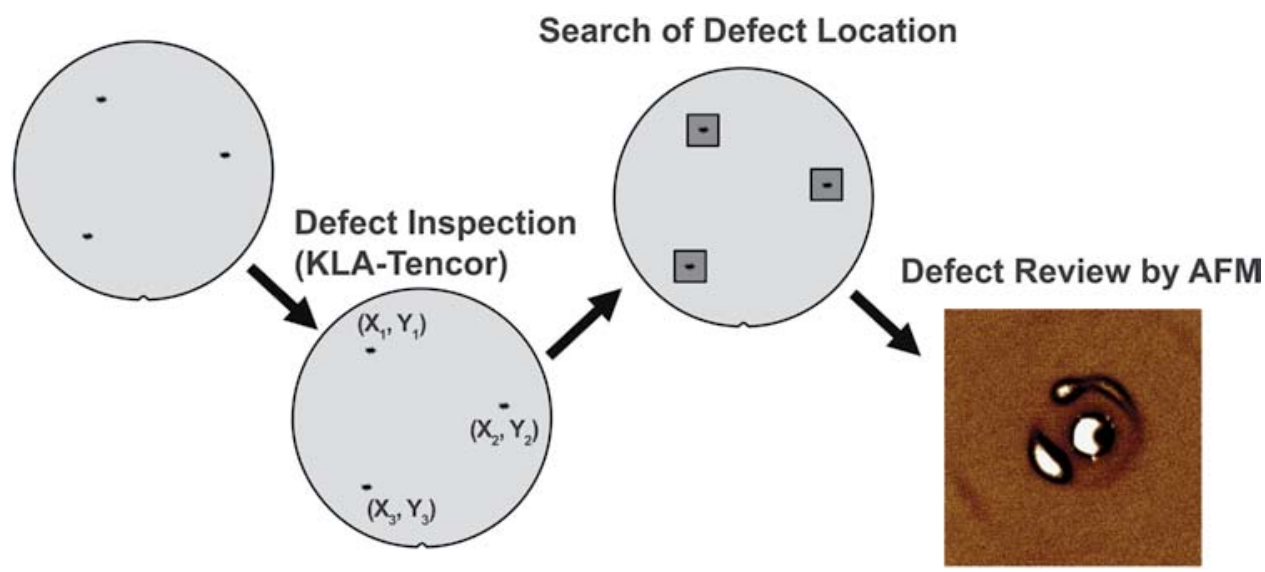

Figure 2: Typical routine of defect review process by manual AFM has a very low throughput-10 defects per day at best. Moreover, the tip cost can run high if one uses a destructive scan mode such as tapping. 


\section{Now is the Time}

Founded in 1991, TESCAN is becoming an industry leader by designing quality products, creating innovative solutions, and listening to our customers' needs.

- Thermionic Scanning Electron Microscopes - Field Emission Scanning Electron Microscopes - FIB-SEM Workstations

- Raman Correlative Microscopy

- Mineral Analysis Solutions

- ToF-SIMS

\section{Quality. Reliability. Performance. www.tescan-usa.com}

TESCAN USA Inc. 765 Commonwealth Dr.

Suite 101

Warrendale, PA 15086

Ph: 724-772-7433

Fx: 724-772-7434 
on a review tool. Coordinates from the inspection tool are translated to the review tool, which is used to zoom in and image the details of the defect, revealing its characteristics. The complicated part comes into play when attempting to accurately go near the defect site, in order to image it,

\section{Coarse Alignment}

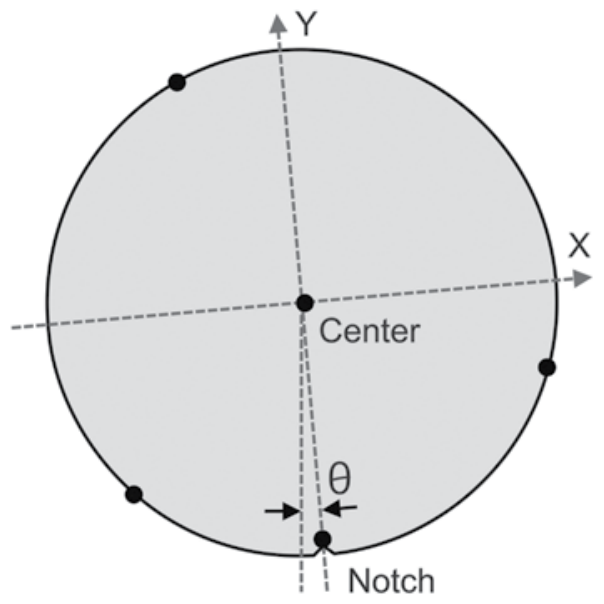

1. Wafer Center Position 2. Rotation Angle $\theta$
Fine Alignment

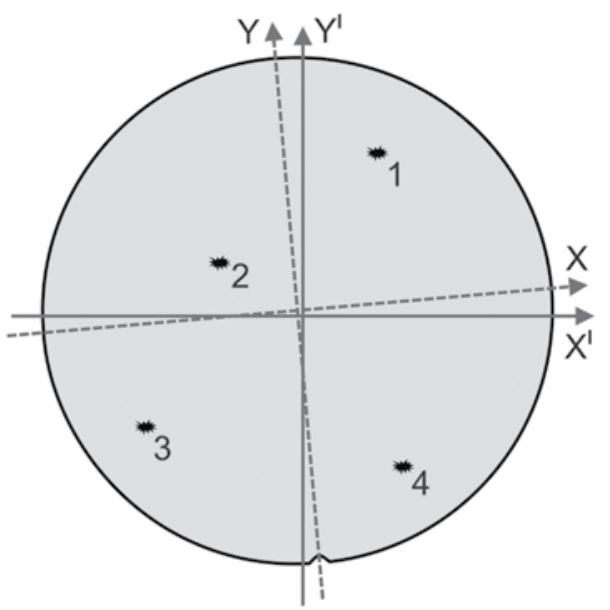

Inspection Tool

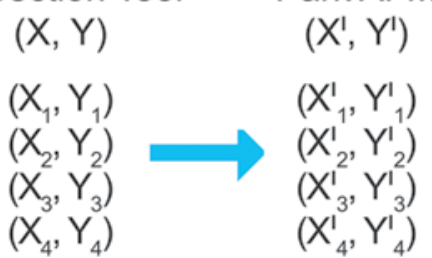

Figure 3: Wafer edge and actual defects are used to orient the wafer and translate the coordinates between the inspection tool and the Park AFM.
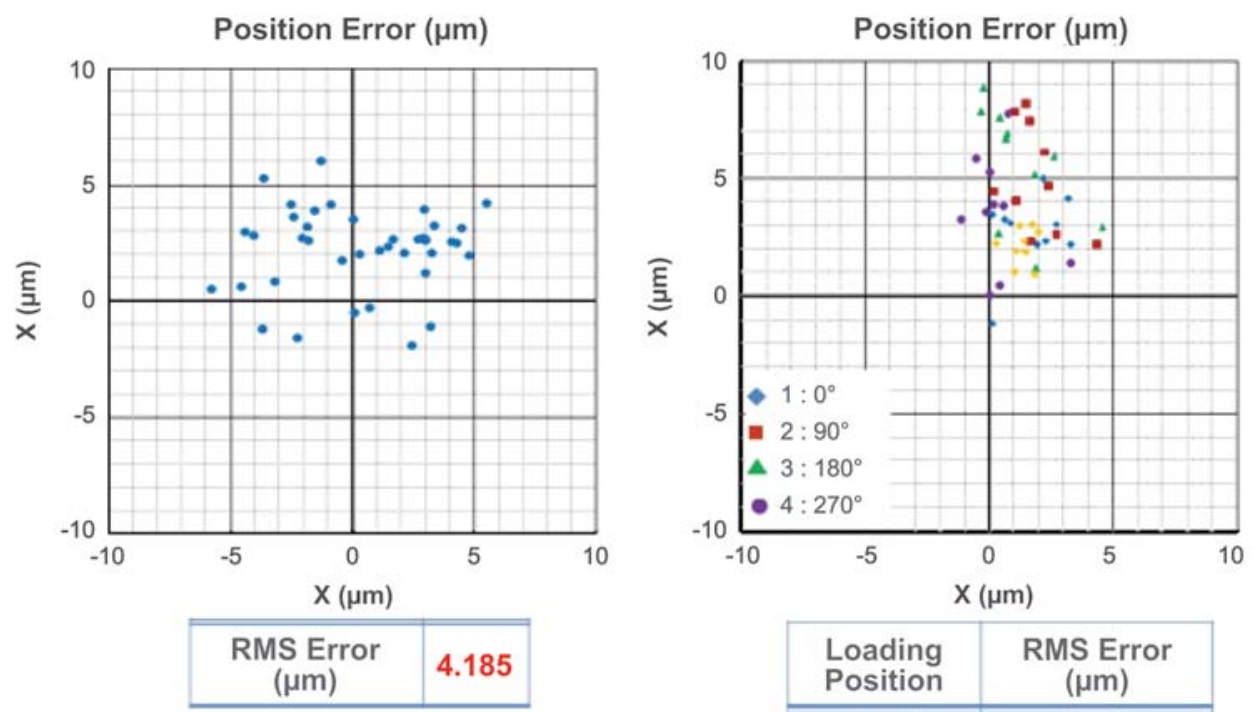

\begin{tabular}{|c|c|}
\hline $\begin{array}{c}\text { Loading } \\
\text { Position }\end{array}$ & $\begin{array}{c}\text { RMS Error } \\
(\mu \mathrm{m})\end{array}$ \\
\hline $1: 0^{\circ}$ & 3.779 \\
\hline $2: 90^{\circ}$ & 5.885 \\
\hline $3: 180^{\circ}$ & 6.423 \\
\hline $4: 270^{\circ}$ & 4.430 \\
\hline \hline Average & 5.129 \\
\hline
\end{tabular}

Figure 4: (a) The positional error of actual defect sites after the automatic stage mapping is small, about $5 \mu \mathrm{m}$ RMS, and (b) remains nearly the same regardless of wafer loading positions. and to do this all in automation. This is because of possible stage mapping errors between the inspection tool and the review tool.

In the case of a patterned wafer, alignment marks are used as reference points to map the wafer-holding stages of the initial inspection tool and the AFM, and the position error from both tools can be accurately estimated (Figure 2). Park's initial success in an ADR AFM solution was implemented in the hard disk industry, using the reference marks inscribed by the light optical inspection tool such as KLA Tencor's Candela series. Using these marks, the defect maps of hard disk media or substrates could be accurately and reliably transferred, and the automated review AFM (Park HDM Series) was able to get near enough to the defect to conduct a survey scan followed by a zoom-in scan to provide the details the user required. As a whole, this solution proved to be effective, increasing the number of defect measurements from 10 per day in a manual method (Figure 2) to 100 per day in a fully automated, unmanned operation.

Transfer of defect map without reference marks. Success in the hard disk drive (HDD) industry resulted in similar requests from the semiconductor industry. Because the cleanness level requirement for the semiconductor industry is much stricter than that of HDD, creating markers on the bare wafers is not possible, hence the need for an ADR process without any reference markings on the bare wafer. Without alignment marks the positions of defects are roughly estimated $[1,2]$. Therefore, additional alignment is required during the defect map transfer [3]. To address this issue, a new method was developed that uses the wafer edge, notch, and large defects, which are visible by LOM, as the reference points. The improved remapping technique, combined with enhanced vision where AFM-based differential frame averaging, is carried out. The new differential frame averaging enhances the contrast of optical view by observing the vision change after displacing the sample position using AFM, which does not require 

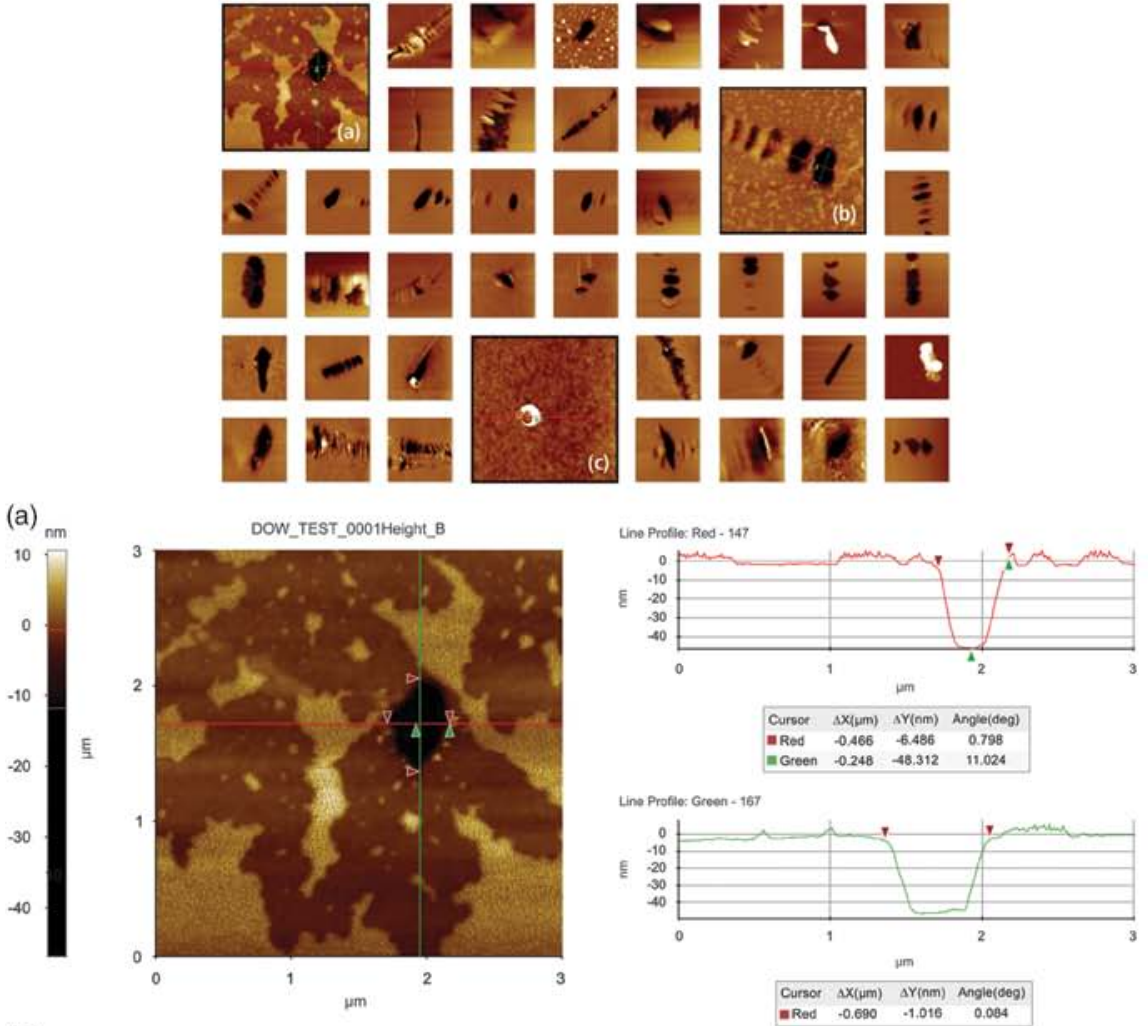

(b) ${ }_{n m} \quad$ DOW_TEST_0008__Height_B
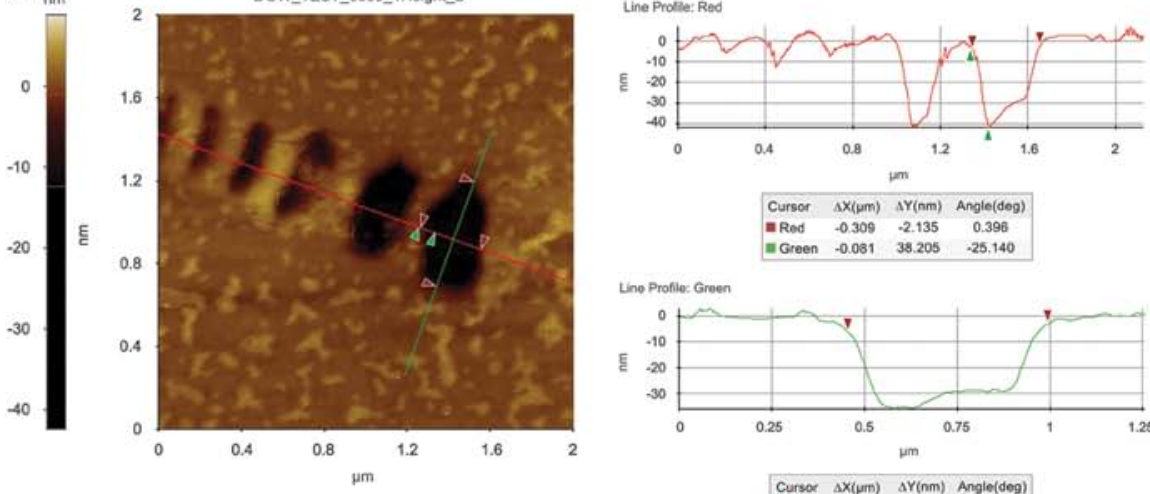

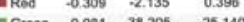

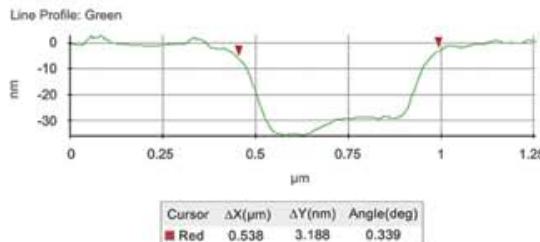

(c)
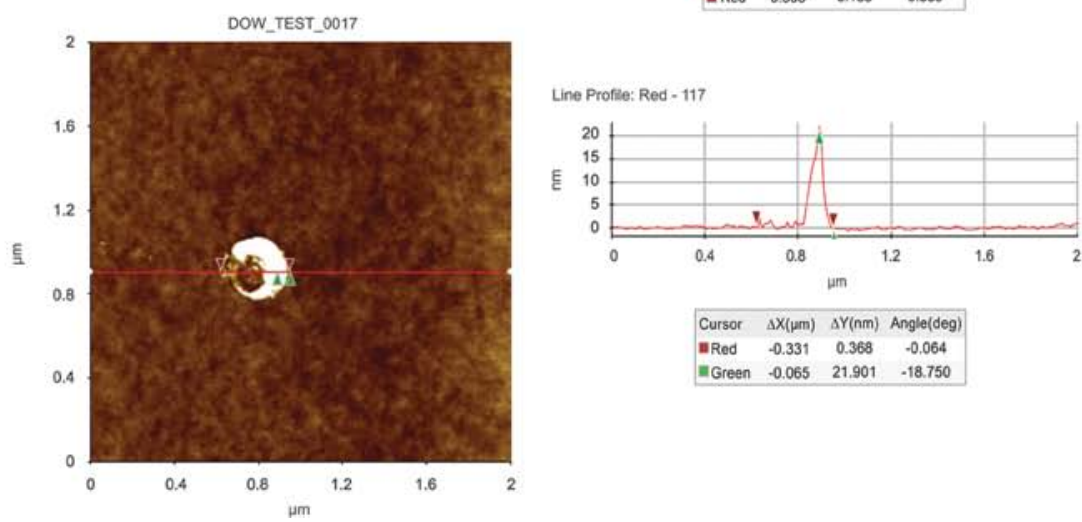

Figure 5: The $300 \mathrm{~mm}$ bare wafer ADR AFM correctly locates all the defect sites identified from an inspection tool and automatically zooms in for detailed imaging. The automatically imaged defects show various type. In (a), a pit-type defect is found and imaged. The horizontal and vertical profiles show a depth of $48 \mathrm{~nm}$ with the size of $0.46 \mu \mathrm{m}$ by $0.69 \mu \mathrm{m}$. In (b) scratch-type defects are shown, probably caused during the polishing process, and they have a depth of about $40 \mathrm{~nm}$ with sizes varying from $0.2 \mu \mathrm{m}$ to $0.5 \mu \mathrm{m}$. In (c) a bump-type defect is imaged with the height of $21.9 \mathrm{~nm}$ and diameter of $130 \mathrm{~nm}$. any reference marker on a bare wafer (Figure 3). Furthermore, it also does not need any separate step to calibrate the stage of the targeted inspection system.

The success rate of the $300 \mathrm{~mm}$ bare wafer ADR depends on various factors, but the two most important ones are the accuracy of stage mapping and the size of initial survey scan. Also, tip life becomes critical as the automation process requires taking hundreds of images uninterrupted. The more accurate the stage mapping, the smaller survey scan size that can be used in finding the defect. The new stage mapping technique improves the accuracy by adopting an advanced remapping algorithm that uses the notch and edges of wafer for coarse alignment to identify the initial defects locations, and further alignment is realized after locating the defects via AFM.

The root-mean-square (RMS) position error of survey scans is less than $5 \mu \mathrm{m}$ regardless of wafer loading positions (see Figure 4). This means that all the defects are located within $\pm 5 \mu \mathrm{m}$ using the defect map provided from a defect inspection tool.

\section{Results}

Using the advanced coordinate translation technique described above, a defect map obtained from a laser-scattering defect inspection tool, such as Surfscan from KLA-Tencor, can be accurately transferred to a $300 \mathrm{~mm}$ AFM system, allowing full automation for high throughput defect imaging. With the new $300 \mathrm{~mm}$ bare-wafer ADR, a typical automated measurement run would go as follows:

(1) A bare wafer is run through a laserscattering defect inspection tool (for example, Surfscan)

(2) Then an operator registers the resulting defect coordinates file, known as KLARF for the Surfscan tool, to the Park NXWafer tool.

(3) Coordinates of the defect map are automatically de-skewed, and the linkage of the two stages is enabled, registering the inspection tool and Park NX-Wafer.

(4) The Park NX-Wafer then runs the ADR on the sample bare wafer.

The following set of data shows the results from the $300 \mathrm{~mm}$ bare wafer ADR with the Park NX-Wafer (Figure 5). Defects as small as $20 \mathrm{~nm}$ in height and $130 \mathrm{~nm}$ in width could be measured with a position error of less than $5 \mu \mathrm{m}$ (Figure 4). 


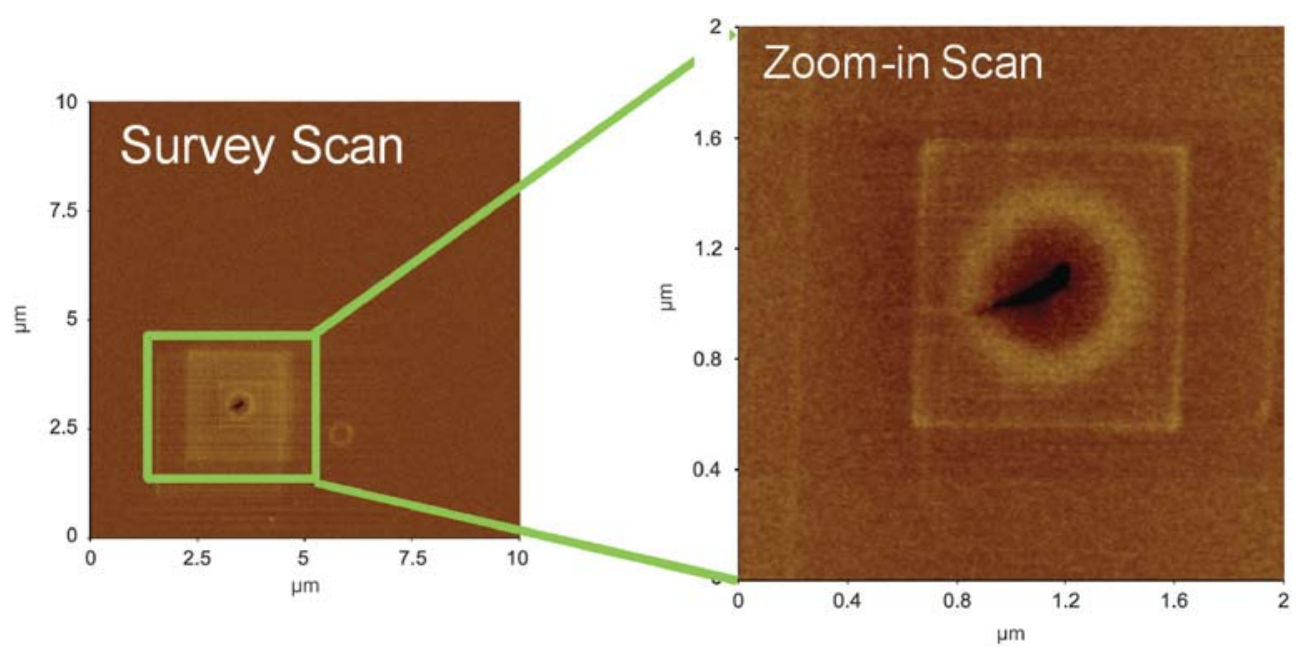

Figure 6: The AFM imaging shows the residual damage left after SEM imaging. first fully automated solution by AFM and can add greatly to existing defect characterization methods, not only for bare wafers but for patterned ones as well.

\section{Conclusion}

Innovative automated AFM solutions for manufacturing control is gaining more importance as device size becomes smaller. The new ADR AFM locates and images defects with a large field of view using AFM-based differential frame averaging. This advancement improves the throughput of defect imaging and monitoring,

Additional measurement with force or electrical mode can be taken on the same defect for the investigation of nanomechanical or electrical properties. Typical success rates are higher than $95 \%$ regardless of the orientation of a wafer. The zoomed-in scans show the detailed morphology of defects, clearly distinguishing various types such as pit, bump, and scratch.

Defect-review SEM can leave destructive square-shaped irradiation marks on defect sites after its run. During the ADR run of some wafer samples, the survey scan and zoom-in scan found residual damage left on defect sites during SEM imaging (Figure 6). The irradiation damage during SEM review process can limit its use in production control.

\section{Discussion}

For automated ADR to work effectively, two important criteria must be met: the AFM system for small-sized defects must operate with ultra-low noise, and the AFM tip must be preserved for automated processing without interruption. The wafer AFM from Park Systems delivers reliability with a system noise level of less than $0.05 \mathrm{~nm}(0.5 \AA)$ RMS measured in zero scan where the tip remains in one position and the $Z$-movement is recorded. It also preserves the tip sharpness through True Non-Contact measurement technology where the large $Z$-feedback bandwidth of up to $10 \mathrm{kHz}$ minimizes tip-sample interaction and effectively prolongs tip life.

By developing a proper algorithm, the AFM can automatically go to each of the defect locations and image the defects in two steps: image a larger, survey scan to refine the defect location, and then image a smaller zoom-in scan to obtain the details of the defect. The key technological challenge here was the accurate transfer and remapping of the defect map from the light optical inspection tool to the AFM stage while reducing the position error of defects to the $X Y$ scan size allowed by AFM, typically less than $50 \mu \mathrm{m} \times 50 \mu \mathrm{m}$. The current system demonstrates a positional error of less than 5 $\mu \mathrm{m}$, which is similar to that of the SEM-based defect review process. The new AFM-based defect review represents the which previously had been done by a time-consuming manual process.

\section{References}

[1] H Ota et al., Hitachi Review 55(2) (2006) 79.

[2] A Nutsch et al., International Symposium on Semiconductor Manufacturing (ISSM) (2007) 1-4.

[3] PD Kinney and YS Uritsky, Proc SPIE In-Line Characterization Techniques for Performance and Yield Enhancement in Microelectronic Manufacturing 3509 (1998) 204-09.

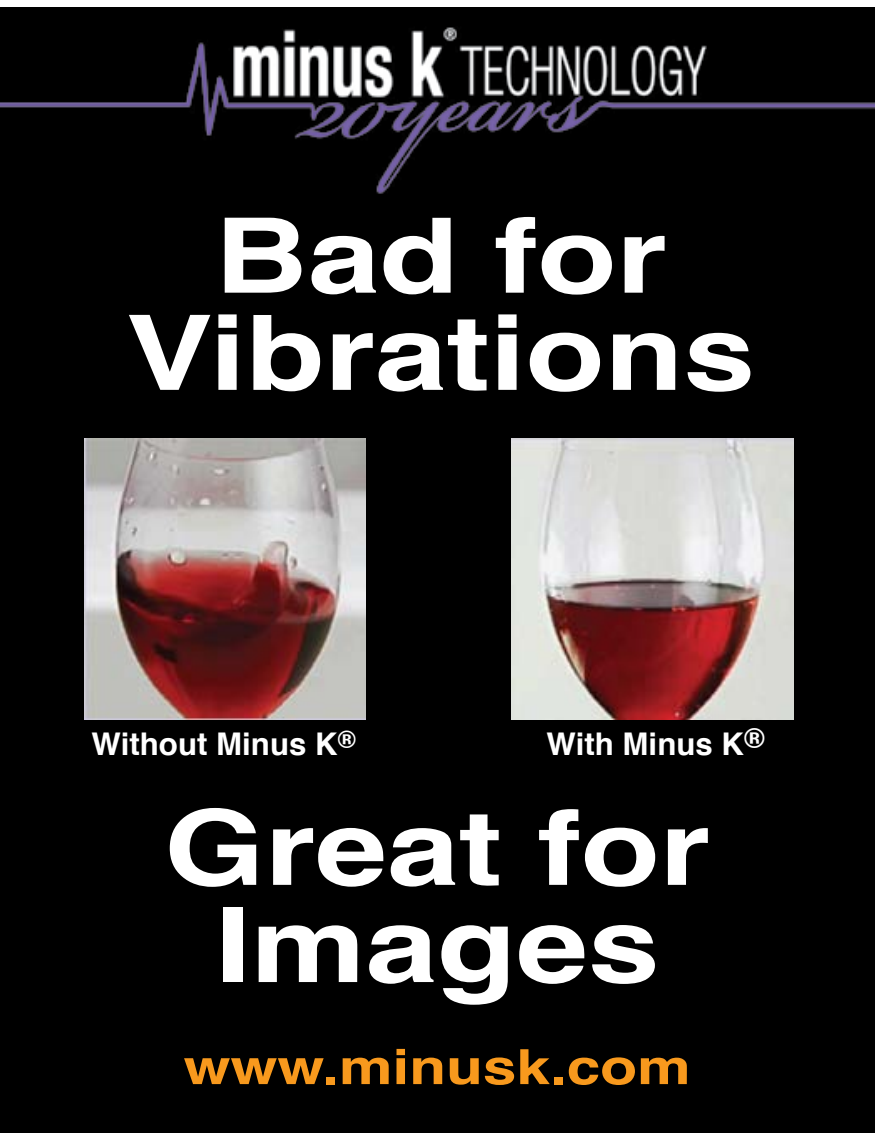




\section{OLYMPUS}

Your Vision, Our Future

\section{See More. Clearly.}
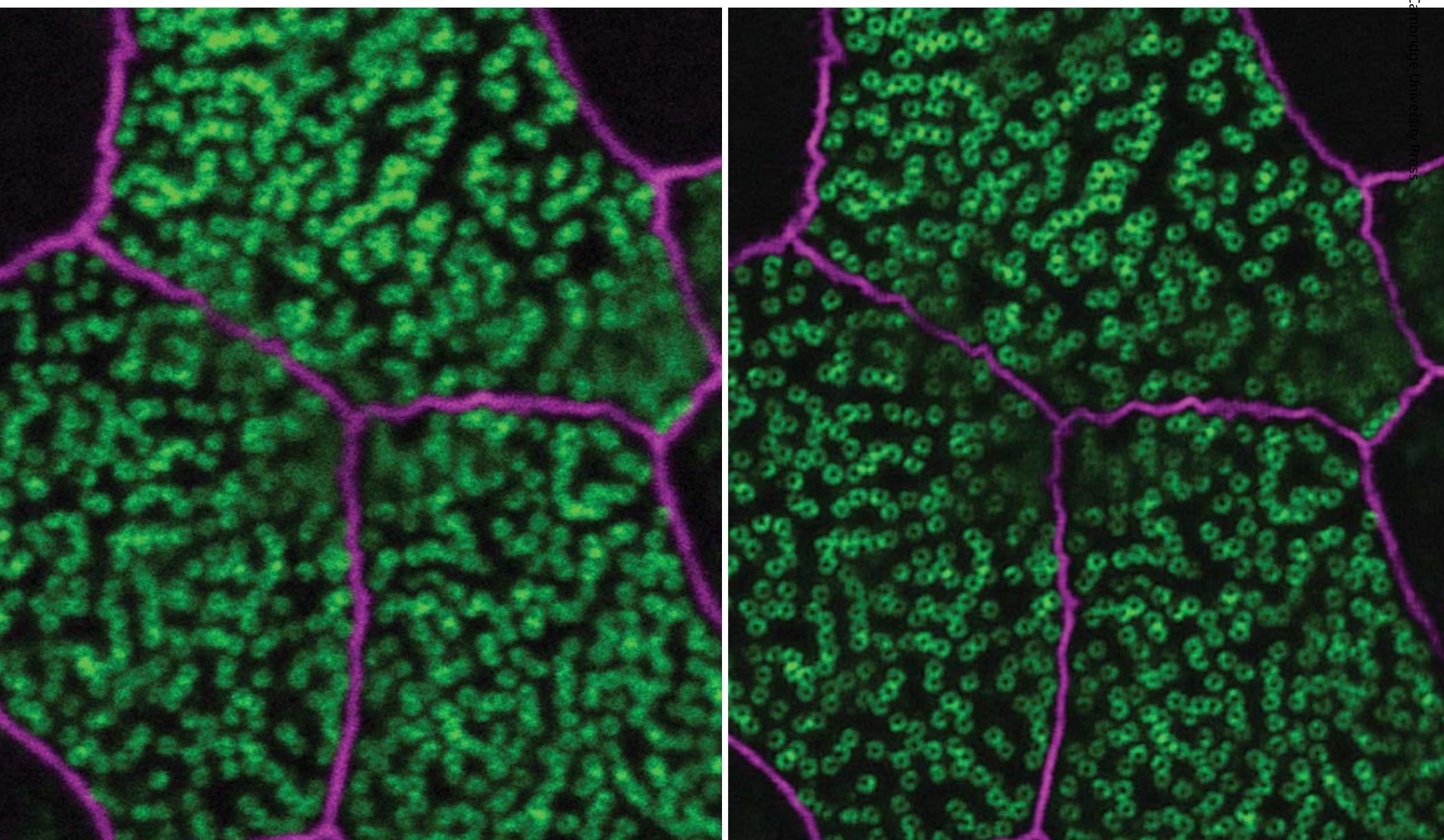

Avoid second guessing the details of your images. At nearly two times the resolution, upgrading any FV1000 or FV1200 confocal system with the new Olympus Super Resolution will help you see those critical fine structures in your samples. Quick, flexible and affordable, FV-OSR allows you to look deeper into your samples - not your budget.

See more. Discover more.

\section{Your Science Matters}

For further information, please visit: YourScienceMatters.com

\section{OLYMPUS}

Life Science, 48 Woerd Avenue, Waltham, MA 02453, 800-446-5967

Sample: Trachea multi-ciliated epithelial cells (Culture) Images courtesy of Graduate School of Frontier Biosciences and Graduate School of Medicine Osaka University: Hatsuho Kanoh, Elisa Herawati, Sachiko Tsukita, Ph.D. 